\title{
Impaired glucose metabolism treatment and carcinogenesis (Review)
}

\author{
ARTUR MATYSZEWSKI ${ }^{1-3}$, ANNA CZARNECKA ${ }^{1}$, MACIEJ KAWECKI $^{1}$, PIOTR KORZEN ${ }^{1}$, \\ ILAN J. SAFIR ${ }^{4}$, WOJCIECH KUKWA ${ }^{5}$ and CEZARY SZCZYLIK ${ }^{1}$ \\ ${ }^{1}$ Department of Oncology, Military Institute of Medicine, Warsaw; \\ ${ }^{2}$ Department of Internal Medicine, Cardiology and Hypertension; ${ }^{3}$ Department of Nephrology and Dialysis Center, \\ L. Rydygier Province Hospital, Suwałki, Poland; ${ }^{4}$ Department of Urology, Emory University School of Medicine, \\ Atlanta, GA, USA; ${ }^{5}$ Department of Otorhinolaryngology, Medical University of Warsaw, Warsaw, Poland
}

Received October 16, 2014; Accepted April 29, 2015

DOI: $10.3892 / \mathrm{ol} .2015 .3324$

\begin{abstract}
Carbohydrate metabolism disorders increase the risk of carcinogenesis. Diabetes mellitus alters numerous physiological processes that may encourage cancer growth. However, treating impaired glucose homeostasis may actually promote neoplasia; maintaining proper glucose plasma concentrations reduces metabolic stresses, however, certain medications may themselves result in oncogenic effects. A number of previous studies have demonstrated that metformin reduces the cancer risk. However, the use of sulfonylurea derivatives correlates with an increased risk of developing a malignancy. Another form of treatment, insulin therapy, involves using various forms of insulin that differ in pharmacodynamics, pharmacokinetics and efficacy. Previous studies have indicated that certain insulin variants also affect the cancer risk. The results from analyses that address the safety of long-lasting insulin types raise the most concern regarding the increased risk of malignancy. Rapid development of novel diabetic medications and their widespread use carries the risk of potentially increased rates of cancer, unnoticeable in limited, randomized, controlled trials. In the present review, the results of clinical and epidemiological studies are evaluated to assess the safety of anti-hyperglycemic medications and their effect on cancer risk and outcomes.
\end{abstract}

Correspondence to: Dr Artur Matyszewski, Department of Oncology, Military Institute of Medicine, 128 Szaserow Street, Warsaw 04-141, Poland

E-mail: artur.matyszewski@gmail.com

Abbreviations: DM, diabetes mellitus; DM2, type 2 DM; IR, insulin receptors; IGF-IR, insulin-like growth factor receptor; GLP-1, glucagon-like peptide-1; GIP, glucose-dependent insulinotropic peptide; DPP-4, dipeptidyl peptidase-4

Key words: carcinogenesis, diabetes, insulin resistance, glargine, metformin

\section{Contents}

1. Introduction

2. Metformin, oral hypoglycemic agents and neoplasia

3. Human insulin, insulin analogues and neoplasia

4. Incretinomimetics and neoplasia

5. Summary

\section{Introduction}

Diabetes mellitus (DM) is a group of metabolic diseases characterized by hyperglycemia resulting from the failure of the production or action of insulin, which is secreted by the $\beta$ cells of the pancreas. Chronic hyperglycemia is associated with damage, disorder and various organ failures, particularly of the eyes, kidneys, nerves, heart and blood vessels. Mortality of diabetic patients is primarily associated with late outcomes: Organ failure (kidneys, heart and vessels), infections and immune system disorders, or the result of untreated hyper- or hypoglycemia $(1,2)$. The data presented by the International Federation of Diabetes (3) indicates that, at present, DM is detected in $>250$ million people worldwide, with $>7$ million new cases diagnosed each year; it is also estimated that, by 2030 , the disease will affect $>400$ million people, of which $90-95 \%$ of all cases will be DM type 2 (DM2), frequently resulting from obesity (3). According to data and predictions at the beginning of the 21st century, the World Health Organization (WHO) estimated that, in 2015, excess weight would affect 2.3 billion people and clinical obesity would manifest in $>700$ million. The incidence of impaired glucose tolerance (IGT) is dynamically increasing in developed countries, with an estimated incidence in the year 2000 of $>470$ million individuals, which is $\sim 9 \%$ of the general population. It is estimated that $50 \%$ of patients exhibiting IGT will develop DM2 within the next 10 years (4).

The initial approach to treating type 2 diabetes mellitus (DM2) and preDM relies on lifestyle changes, including increased exercise and diet modification. If lifestyle interventions are insufficient, the introduction of oral hypogylcemic agents or insulin therapy may be necessary (1). 
According to current DM2 management guidelines (5), metformin treatment should be initiated as early as possible in conjunction with lifestyle modifications. Untreated DM2 typically requires the initiation of exogenous insulin treatment after $\sim 10$ years. It has previously been demonstrated that long-term treatment with oral hypoglycemic drugs or exogenous insulin results in specific adverse effects, including an increased risk of malignancy in the context of a chronic inflammatory state (2). In the present review, the oncogenic potential of metformin, insulin analogues and incretinomimetics in the treatment of DM2 are discussed (1).

\section{Metformin, oral hypoglycemic agents and neoplasia}

Metformin is currently the first-line treatment for DM2 in overweight and obese patients. Sulfonylurea derivatives are an alternative treatment for patients of normal weight and for cases where there is metformin intolerance. Acarbose, repaglinide, glitazar, dipeptidyl peptidase-4 inhibitors and glucagon-like peptide-1 analogues are used less frequently $(1,5,6)$.

Several epidemiological studies have demonstrated that metformin, as a monotherapy or in combination with other hypoglycemic agents, reduces the risk of malignancy among patients with impaired glucose metabolism (7). A retrospective analysis of almost 63,000 patients with DM2, diagnosed after the age of 40 in an English primary healthcare setting, demonstrated an increased incidence of certain types of cancer (pancreas, colon, breast and prostate) among patients receiving sulfonylurea derivatives or insulin, when compared with patients taking metformin. The associated increased cancer risk was $36 \%$ for sulfonylurea and $42 \%$ for insulin (8). Concurrent metformin treatment reduced the aforementioned increased risks of sulfonylurea derivative or insulin regimens. This phenomenon was most significant in the insulin and metformin co-therapy cohort, with a risk-reduction of $46 \%$. A case-control study evaluating patients with DM and pancreatic adenocarcinoma, performed at the MD Anderson Cancer Center (Houston, TX, USA), demonstrated a significant $62 \%$ risk-reduction of adenocarcinoma development among patients treated with metformin (9). By contrast, patients treated with sulfonylurea derivatives or an insulin variant exhibited an risk of developing pancreatic adenocarcinoma that was increased by 2.5- and 5-fold, respectively. A reduced risk of neoplasia development was also associated with metformin usage in another observational cohort study of 4,000 Scottish patients with a diagnosis of DM2 and 9 years of follow-up data (10). The study group consisted of a group of diabetic patients who received metformin treatment and a control group of diabetic patients who did not receive metformin. The metformin-treated group demonstrated a $37 \%$ reduction in the relative cancer risk and an even greater $54 \%$ reduction in the absolute cancer risk when adjusted for co-variables, including age, body mass index and history of tobacco use.

A previous study that analyzed the risk of cancer-associated mortality and DM treatment observed a similar pattern to the aforementioned result (7). Outcomes of the prospective ZODIAC study, which included 1,300 patients with DM2 treated in DM clinics in Holland, demonstrated a 57\% reduction in cancer-associated mortality among patients treated with metformin when compared with those treated without metformin (11). Additionally, the study demonstrated a dose-dependent correlation between metformin intake and the risk of developing malignancy. A retrospective analysis from Canada compared cancer-associated mortality between patients treated with metformin and sulfonylurea derivatives, and between those treated with or without insulin $(12,13)$. The study demonstrated that the relative risk of cancer-associated mortality was significantly increased among patients treated with sulfonylurea derivatives when compared with the metformin group. The study demonstrated that insulin monotherapy significantly increased the risk of cancer-associated mortality and that this increase was proportional to the total daily dose of insulin.

Numerous studies have indicated that there may be a correlation between metformin use and a reduced incidence of neoplasia development (14-19). In vitro studies and animal models support this finding (14). The clinically observed protective action of metformin likely results from the suppression of intracellular signaling pathways that normally transduce activating signals from insulin receptors (IRs) and insulin-like growth factor receptors (IGF-IRs) (15-18). Conversely, hypoglycemic agents that act by increasing plasma concentrations of insulin, endogenously, as in the case of sulfonylurea derivatives, or exogenously, as in the case of insulin therapy, may result in increased cancer incidence by stimulating the aforementioned signal transduction pathways.

\section{Human insulin, insulin analogues and neoplasia}

Insulin deficiency, either with or without insulin resistance, requires treatment with exogenous insulin (7). Achievements in genetic engineering have enabled the industrial-scale production of insulin that has a similar structure to endogenous human insulin and exhibits analogous properties. Furthermore, genetic engineers have altered the molecular structure of insulin and its biochemical properties, subsequently creating insulin analogues. Alterations in the composition and amino acid sequences of insulin polypeptide chains result in different pharmacokinetic and pharmacodynamic properties, and change the binding affinity of the molecule for IRs and IGF-IRs (7). Increased affinity towards these receptors may amplify intracellular signaling associated with insulin and insulin-like growth factors, and concomitantly induce mechanisms that promote carcinogenesis. In light of these proposed tumorigenesis pathways, insulin analogues have been increasingly studied for their oncogenic properties. Kurtzhals et al (20) compared the in vitro oncogenic properties of human insulin, commercial insulin analogues (aspart, lispro, glargine and detemir) and B10Asp; B10Asp is an insulin analog with known carcinogenic potential, as previously demonstrated by in vitro studies and animal models (19). A previous study analyzed the affinities of different molecules to the IRs and IGF-IRs, along with analyzing the metabolic and mitogenic effects of the molecules on cell cultures (20). The properties exhibited by rapid-acting insulin analogues, including aspart and lispro, were comparable to insulin; however, long-acting insulin analogues, including detemir and glargine, differed significantly from insulin in their properties. Detemir demonstrated a reduced affinity towards the two receptor types and 


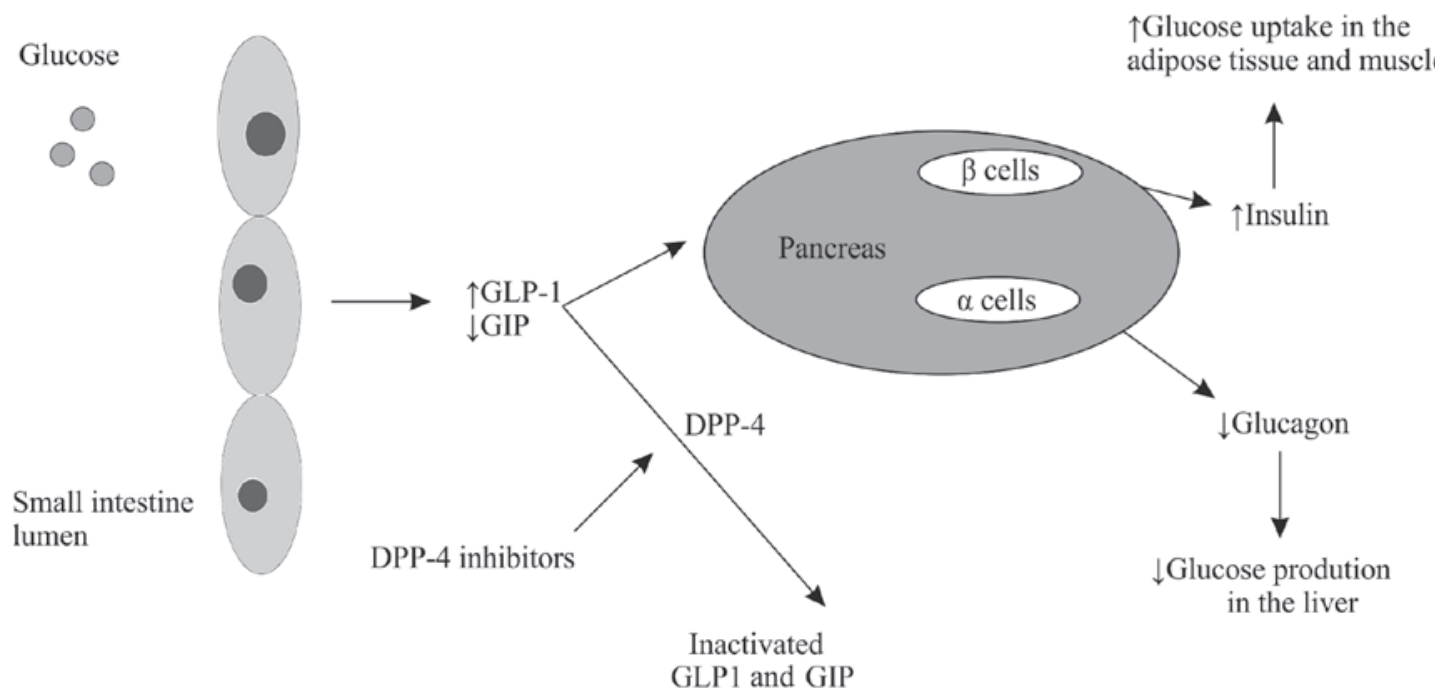

Figure 1. Scheme of incretin axis. GLP-1, glucagon-like peptide-1; GIP, glucose-dependent insulinotropic peptide; DPP-4, dipeptidyl peptidase 4.

demonstrated lesser metabolic and mitogenic effects on cell cultures. Glargine, by contrast, demonstrated a 6 -fold stronger affinity towards IGF-IR and an 8-fold greater mitogenic effect on cell cultures, characteristics more similar to B10Asp than to human insulin (20).

Sciacca et al (21) compared the signal transduction activity of rapid-acting insulin analogues (aspart, lispro, and glulisine) and long-acting insulin analogues (detemir and glargine) with human insulin, insulin-like growth factor 1 and the insulin analog B10Asp on cells in culture. The cells used in the study were mice fibroblasts that expressed IR types A and B and IGF-IR. As in the prior study by Kurtzhal et al (20), the characteristics of the rapid-acting insulin analogues were similar to those of human insulin. The long-acting insulin analogues, however, significantly increased the intracellular signaling cascade dependent on type A IR and IGF-IR, and therefore increased cellular proliferation. However, other than B10Asp, which acts primarily via the type A IR, none of the evaluated insulin analogues results in the oncogenic transformation of the cell lines.

Mayer and Chantelau (22) investigated the effects of human insulin and insulin analogues on breast cancer cells. Breast cancer cells were isolated from patients with type $1 \mathrm{DM}$, and were treated with human insulin and insulin analogues. The cancer cells were subsequently incubated with blood serum that lacked peptide $\mathrm{C}$. The mitogenic potential of the serum was increased by $11 \%$ when it contained glargine compared with when it contained human insulin $(\mathrm{P}=0.005)$. The mitogenic potential of the serum containing detemir was slightly reduced compared with that containing human insulin $(\sim 1 \%)$.

Previous epidemiological analyses studying the oncogenic potential of insulin analogues have been inconclusive. However, concerning findings were reported from a German retrospective analysis of $>127,000$ patients (23). The study compared aspart, lispro or glargine as monotherapy versus standard human insulin and analyzed the subsequent development of malignancy. Multiple insulin analogues demonstrated a positive correlation between daily doses of insulin analogues and the risk of developing neoplasia. However, following correction for dose differences and multiple population variables, glargine was the only insulin analogue that demonstrated a statistically significant correlation with an increased risk of neoplasia development. By contrast, a Swedish study of 114,000 patient medical histories demonstrated no statistically significant differences in the cancer risk between glargine and other insulin analogues (24). A retrospective review of 36,000 Scottish patients also reported no increase in the cancer risk among glargine users (25). A meta-analysis of 31 randomized studies, undertaken by Sanofi (Surrey, UK), a company that manufactures insulin analogues, compared glargine usage with different DM treatment regimens and demonstrated no increase in the cancer risk (26,27). Furthermore, treating patients with glargine resulted in a $10 \%$ reduction in the relative-risk of cancer incidence compared with patients that received different interventions. Nonetheless, funding sources and institutional biases must be considered when considering findings from this meta-analysis.

The risk of carcinogenesis following detemir treatment has also been evaluated in multiple studies. In vitro studies demonstrated that there was no additional risk of an oncogenic transformation associated with detemir treatment (28). Human studies involving diabetics treated with detemir demonstrated similar results. A meta-analysis of 16 studies comparing detemir with neutral protamine Hagedorn and human insulin, in addition to 5 studies that compared detemir with glargine, demonstrated a reduced risk of developing neoplasia in detemir users (28). Compared with detemir, studies continue to demonstrate the increased oncogenic potential of glargine. A long-term, randomized, control trial of 1,340 patients with DM2 dependent on insulin therapy monitored patients for a mean time period of 76 months (29). The study matched every patient diagnosed with cancer in this study population $(n=112)$ with a control patient who did not develop cancer, but had a similar follow-up time period, risk factors and general characteristics. Patients diagnosed with cancer received an increased mean daily dose of glargine compared with the control group: $0.24 \mathrm{IU} / \mathrm{kg}$ of body weight vs. $0.16 \mathrm{IU} / \mathrm{kg}$ of body weight, respectively $(\mathrm{P}=0.036)$. Cases 
that were diagnosed with cancer correlated strongly with glargine daily doses $\geq 0.3 \mathrm{IU} / \mathrm{kg}$ of body weight. This correlation remained following correction for concomitant diseases, other insulin doses or concomitant metformin usage. The absolute risk of increased cancer incidence was increased 5.5-fold when compared with the control group $(\mathrm{P}=0.001)$. Statistical analysis demonstrated no correlation between the increased risk of developing cancer and the daily doses of human insulin and insulin analogues other than glargine. Questions regarding the safety of glargine have been the driving force behind subsequent studies comparing different insulin analogues and studies on novel insulin analogues, such as degludec (30).

It is notable that the United States Food and Drug Administration (FDA) has warnings regarding the use of inhalational insulin due to its possible increased cancer risk, specifically for lung cancer. In a study comparing inhalational insulin with the typical subcutaneous route of administration, 6 cases of non-small cell lung cancer were reported in the inhalational insulin group compared with 1 reported case in the control group (31). Given these findings, different routes of insulin administration may pose an increased risk of cancer and warrant further investigation.

The overall scientific literature regarding the effects of the myriad of diabetic treatment regimens and modalities remain limited. At present, there is no consensus on the safety and oncogenic potential of insulin analogues; however, the positive role of metformin in DM management and its protective effects on cancer development, as monotherapy and in combination with other hypoglycemic drugs, can confidently be affirmed (6-11,14-18). By contrast, drugs that act via increasing endogenous insulin concentrations, in addition to exogenous insulin itself, may increase the cancer risk (7-9,12,13,32-44). Despite in vitro studies indicating that glargine may stimulate carcinogenesis, the available evidence from epidemiological analyses indicates it is premature to advocate a causative association between glargine use and the increased risk of developing cancer. The possibility that individual differences in glargine metabolism or the use of atypically high doses of insulin analogues may increase the cancer risk cannot be excluded (20-27,29-30). Expert recommendations from multiple professional committees (FDA, EASD, EMEA, IDF and ADA) state that there is no reason for limiting glargine use in the general DM population. However, in cases of existing proliferative diseases, such as cancer, or in patient populations with a known higher risk of cancer development, such as women with BRCA1 or BRCA2 mutations, different insulin analogues other than glargine should be considered as the first choice treatment (45-47).

\section{Incretinomimetics and neoplasia}

One of the most recent medication classes developed for DM2 treatment are the incretinomimetics (Fig. 1) (48). This drug class has been used in the USA since 2006 and in Europe since 2008. In the pathogenesis of DM2, rising insulin resistance is accompanied by the concomitant dysfunction of pancreatic $\beta$ cells. It is hypothesized that $\beta$ cell dysfunction may be a result of the abnormal regulation of a class of gastrointestinal hormones, termed incretins. Reduction of glucagon-like peptide-1 (GLP-1) secretion and simultaneous impairment of glucose-dependent insulinotropic peptide (GIP) function, despite appropriate hormonal concentrations, results in the loss of normal insulin secretion patterns observed during the second phase of insulin release. GLP-1 analogues and GLP-1 receptor agonists, in addition to inhibitors of dipeptidyl peptidase-4 (DPP-4), an enzyme that degrades incretins, have been intensively studied as potential novel drug therapies for DM2 (48). When administered to DM2 patients, GLP-1 demonstrates superiority to GIP, as it improves the early and late phases of insulin release. There are two biochemical approaches in treating DM2 that utilize the incretin hormonal pathway. One approach involves the stimulation of GLP-1 receptors with a receptor agonist, such as exenatide, or a GLP-1 analog, such as liraglutide. The second approach involves blocking DDP-4 by specific inhibitors, including sitagliptin, vildagliptin and saxagliptin. The two approaches reduce the blood glucose concentration, improve metabolic homeostasis, and improve quality of life through improved diabetic control. GLP-1 targeted therapy has also been demonstrated to reduce body weight (49).

Long-term preclinical trials on animal models demonstrated a lack of carcinogenic potential of DPP-4 inhibitors, despite such indications from in vitro studies. In theory, DPP-4 may promote tumor progression due to the fact that the inhibited enzyme is a suppressor protein (33-35). A number of previous studies have indicated that there is a correlation between GLP-1 analogues and DPP-4 inhibitors and an increased risk of medullary thyroid cancer and a more aggressive course of multiple endocrine neoplasia type 2 . This effect may be due to increased GLP-1 receptor-dependent intracellular signaling transduction pathways (35-40,50). Another possibility is due to a mutation in the proto-oncogene RET, although this has not been confirmed (41). Changes in DPP-4 expression levels have been identified in hematological malignancies and various types of cancer, including cancers of the ovary, uterus and prostate, non-small cell lung cancer, and neuroendocrine tumors of the gut and pancreas (42). Loss of DPP-4 activity has been shown to correlate with a more aggressive malignancy and an earlier metastatic presentation (50). By contrast, expression of GLP-1 receptors in colon cancer presented as a positive prognostic factor for treatment efficacy $(43,44)$.

Further studies on incretinomimetics and carcinogenesis are required, since the available data is limited compared with older hypoglycemic agents. The majority of cited authors advise moderate optimism and caution when using incretinomimetics in patients with an increased baseline risk of cancer and in patients with known malignancy. Future investigations may aid in the evaluation of the oncogenic safety of GLP-1 agonists and DPP-4 inhibitors. Until then, clinicians should attentively consider every case individually, weighing the risks and benefits, prior to initiating treatment with incretinomimetics.

\section{Summary}

Metformin use in patients with preDM and DM2 significantly reduces the risk of developing malignancy and prolongs survival rates. Sulfonylurea derivatives and certain insulin 
analogues, primarily high daily doses of glargine, may increase the risk of developing malignancy; however, further studies are warranted. At present, expert recommendations state that there is no reason to delay initiation of insulin analogues for DM2 treatment in the typical diabetic population; however, attention should be given when using glargine or incretinomimetics in patients with an increased cancer risk and in patients with a known malignancy. Nonetheless, the appropriate treatment of preDM and DM2, beginning with lifestyle modifications and possibly starting metformin treatment, is the best initial strategy to reduce the cancer risk.

\section{Acknowledgements}

This study has been supported by the National Science Centre (grant no. UMO-2012/05/D/NZ5/01844).

\section{References}

1. Sieradzki JC: Diabetes mellitus. In: Szczeklik's Internal Medicine. Szczeklik A and Gajewski P (eds). Medycyna Praktyczna, Kraków, pp1353-1402, 2014 (In Polish).

2. Matyszewski A, Czarnecka A, Solarek W, et al: Molecular basis of carcinogenesis in diabetic patients (Review). Int J Oncol 46: 1435-1443, 2015.

3. World Health Organization: Obesity and overweight. http:// www.who.int/mediacentre/factsheets/fs311/en/ (Accessed November 30, 2010).

4. International Diabetes Federation: Diabetes Atlas. 6th edition. http://www.idf.org/diabetesatlas/content/europe $\% 3 b \% 20$ (Accessed November 30, 2010).

5. Polish Diabetes Association: Clinical recommendation concerning the management in patients with diabetes in 2014. Clinical Diabetology 3 (Suppl A), 2014. http://www.cukrzyca. info.pl/zalecenia_kliniczne/zalecenie_kliniczne_dotyczace postepowania_u_chorych_na_cukrzyce_2014. (In Polish; Accessed September 30, 2014).

6. Inzucchi SE, Bergenstal RM, Buse JB, et al; American Diabetes Association (ADA); European Association for the Study of Diabetes (EASD): Management of hyperglycaemia in type 2 diabetes: A patient-centered approach: Position statement of the American Diabetes Association (ADA) and the European Association for the Study of Diabetes (EASD). Diabetes Care 35: 1364-1379, 2012

7. Piątkiewicz P and Czech A: Glucose metabolism disorders and the risk of cancer. Arch Immunol Ther Exp (Warsz) 59: 215-230, 2011.

8. Currie CJ, Poole CD and Gale EA: The influence of glucose-lowering therapies on cancer risk in type 2 diabetes. Diabetologia 52: 1766-1777, 2009.

9. Li D, Yeung SC, Hassan MM, Konopleva M and Abbruzzese JL: Antidiabetic therapies affect risk of pancreatic cancer. Gastroenterology 137: 482-488, 2009.

10. Libby G, Donnelly LA, Donnan PT, Alessi DR, Morris AD and Evans JM: New users of metformin are at low risk of incident cancer: A cohort study among people with type 2 diabetes. Diabetes Care 32: 1620-1625, 2009.

11. Landman GW, Kleefstra N, van Hateren KJ, Groenier KH, Gans RO and Bilo HJ: Metformin associated with lower cancer mortality in type 2 diabetes: ZODIAC-16. Diabetes Care 33: 322-326, 2010

12. Bowker SL, Majumdar SR, Veugelers $P$ and Johnson JA: Increased cancer-related mortality for patients with type 2 diabetes who use sulfonylureas or insulin. Diabetes Care 29: 254-258, 2006.

13. Bowker SL, Yasui Y, Veugelers $P$ and Johnson JA: Glucose-lowering agents and cancer mortality rates in type 2 diabetes: Assessing effects of time-varying exposure. Diabetologia 53: 1631-1637, 2010.

14. Chong CR and Chabner BA: Mysterious metformin. Oncologist 14: 1178-1181, 2009.

15. Buzzai M, Jones RG, Amaravadi RK, et al: Systemic treatment with the antidiabetic drug metformin selectively impairs p53-deficient tumor cell growth. Cancer Res 67: 6745-6752,2007.
16. Kisfalvi K, Eibl G, Sinnett-Smith J and Rozengurt E: Metformin disrupts crosstalk between $\mathrm{G}$ protein-coupled receptor and insulin receptor signaling systems and inhibits pancreatic cancer growth. Cancer Res 69: 6539-6545, 2009.

17. Gonzalez-Angulo AM and Meric-Bernstam F: Metformin: A therapeutic opportunity in breast cancer. Clin Cancer Res 16: 1695-1700, 2010.

18. Rozengurt E, Sinnett-Smith J and Kisfalvi K: Crosstalk between insulin/insulin-like growth factor-1 receptors and $\mathrm{G}$ protein-coupled receptor signaling systems: a novel target for the antidiabetic drug metformin in pancreatic cancer. Clin Cancer Res 16: 2505-2511, 2010.

19. Jorgensen LN, Dideriksen LH and Drejer K: Carcinogenic Effect Of The Human Insulin Analog B10Asp In Female Rats. Diabetologia 35: A3, 1992.

20. Kurtzhals P, Schäffer L, Sørensen A, et al: Correlations of receptor binding and metabolic and mitogenic potencies of insulin analogs designed for clinical use. Diabetes 49: 999-1005, 2000.

21. Sciacca L, Cassarino MF, Genua M, et al: Insulin analogues differently activate insulin receptor isoforms and post-receptor signalling. Diabetologia 53: 1743-1753, 2010.

22. Mayer D and Chantelau E: Treatment with insulin glargine (Lantus) increases the proliferative potency of the serum of patients with type-1 diabetes: a pilot study on MCF-7 breast cancer cells. Arch Physiol Biochem 116: 73-78, 2010

23. Hemkens LG, Grouven U, Bender R, et al: Risk of malignancies in patients with diabetes treated with human insulin or insulin analogues: A cohort study. Diabetologia 52: 1732-1744, 2009.

24. Jonasson JM, Ljung R, Talbäck M, Haglund B, Gudbjörnsdòttir S and Steineck G: Insulin glargine use and short-term incidence of malignancies - a population-based follow-up study in Sweden. Diabetologia 52: 1745-1754, 2009.

25. Colhoun HM, SDRN Epidemiology Group: Use of insulin glargine and cancer incidence in Scotland: A study from the Scottish Diabetes Research Network Epidemiology Group. Diabetologia 52: $1755-1765,2009$.

26. Home PD and Lagarenne P: Combined randomised controlled trial experience of malignancies in studies using insulin glargine. Diabetologia 52: 2499-2506, 2009.

27. Rosenstock J,Fonseca V, McGill JB, et al: Similar risk of malignancy with insulin glargine and neutral protamine Hagedorn (NPH) insulin in patients with type 2 diabetes: Findings from a 5 year randomised, open-label study. Diabetologia 52: 1971-1973, 2009.

28. Dejgaard A, Lynggaard H, Råstam J and Krogsgaard Thomsen M: No evidence of increased risk of malignancies in patients with diabetes treated with insulin detemir: A meta-analysis. Diabetologia 52: 2507-2512, 2009.

29. Mannucci E, Monami M, Balzi D, et al: Doses of insulin and its analogues and cancer occurrence in insulin-treated type 2 diabetic patients. Diabetes Care 33: 1997-2003, 2010.

30. Heise T, Tack CJ, Cuddihy R, et al: A new-generation ultra-long-acting basal insulin with a bolus boost compared with insulin glargine in insulin-naive people with type 2 diabetes: A randomized, controlled trial. Diabetes Care 34: 669-674, 2011.

31. U.S. Food and Drug Administration: Safety Alerts for Human Medical Products - Exubera (insulin human rDNA origin) Inhalation Powder. http://www.fda.gov/Safety/MedWatch/ SafetyInformation/SafetyAlertsforHumanMedicalProducts/ ucm085319.htm (Accessed September 30, 2014).

32. Pro B and Dang NH: CD26/dipeptidyl peptidase IV and its role in cancer. Histol Histopathol 19: 1345-1351, 2004.

33. Wesley UV, McGroarty M and Homoyouni A: Dipeptidyl peptidase inhibits malignant phenotype of prostate cancer cells by blocking basic fibroblast growth factor signaling pathway. Cancer Res 65: 1325-1334, 2005

34. Masur K, Schwartz F, Entschladen F, Niggemann B and Zaenker KS: DPPIV inhibitors extend GLP-2 mediated tumour promoting effects on intestinal cancer cells. Regul Pept 137: 147-155, 2006.

35. Duntas LH: Clinical comments related to medullary thyroid cancer diagnosis and management. Thyroid Res 6 (Suppl 1): S6, 2013.

36. Chiu WY, Shih SR and Tseng CH: A review on the association between glucagon-like peptide-1 receptor agonists and thyroid cancer. Exp Diabetes Res 2012: 924168, 2012.

37. Vangoitsenhoven R, Mathieu C and Van der Schueren B: GLP1 and cancer: Friend or foe? Endocr Relat Cancer 19: F77-F88, 2012.

38. Arora S, Mehrotra A and Gulati SC: Incretins and thiazolidinediones in glucose homeostasis and cancer: Role of common polymorphisms. Cancer Lett 323: 128-134, 2012.

39. Gier B, Butler PC, Lai CK, Kirakossian D, DeNicola MM and Yeh MW: Glucagon like peptide-1 receptor expression in the human thyroid gland. J Clin Endocrinol Metab 97: 121-131, 2012. 
40. Waser B, Beetschen K, Pellegata NS and Reubi JC: Incretin receptors in non-neoplastic and neoplastic thyroid $C$ cells in rodents and humans: relevance for incretin-based diabetes therapy. Neuroendocrinology 94: 291-301, 2011.

41. Madsen LW, Knauf JA, Gotfredsen C, et al: GLP-1 receptor agonists and the thyroid: C-cell effects in mice are mediated via the GLP-1 receptor and not associated with RET activation. Endocrinology 153: 1538-1547, 2012.

42. Waser B, Rehmann R, Sanchez C, Fourmy D and Reubi JC: Glucose-dependent insulinotropic polypeptide receptors in most gastroenteropancreatic and bronchial neuroendocrine tumors. J Clin Endocrinol Metab 97: 482-488, 2012.

43. Kissow H, Hartmann B, Holst JJ, et al: Glucagon-like peptide-1 (GLP-1) receptor agonism or DPP-4 inhibition does not accelerate neoplasia in carcinogen treated mice. Regul Pept 179: 91-100, 2012

44. Koehler JA, Kain T and Drucker DJ: Glucagon-like peptide-1 receptor activation inhibits growth and augments apoptosis in murine CT26 colon cancer cells. Endocrinology 152 3362-3372, 2011.

45. European Association for the Study of Diabetes: Lantus insulin: a possible link with cancer which requires further investigation http://webcast.easd.org/press/glargine/glargine.htm (Accessed September 30, 2014)
46. International Diabetes Federation: Statement from the International Diabetes Federation Related to Studies Published in Diabetologia Suggesting Possible Link Between Insulin Glargine and Cancer. http://www.idf.org/statement-interna tional-diabetes-federation-related-studies-published-diabetologia-suggesting-possib (Accessed September 30, 2014).

47. American Diabetes Association: Studies Find No Increase in Cancer Risk from Insulin Glargine. http://www.diabetes.org/ newsroom/press-releases/2012/sci-sessions-insulin-cancer.html (Accessed September 30, 2014).

48. Matuszek B, Lenart-Lipińska $M$ and Nowakowski A: Incretin hormones in the treatment of type 2 diabetes. Part II. Incretins - new possibilities for pharmacotherapy of type 2 diabetes. Endokrynol Pol 59: 322-329, 2008 (In Polish).

49. Znaniecka M, Rutkowska J and Bandurska-Stankiewicz E: A new direction in the treatment of diabetes - inncretinomimetics and DPP-4 inhibitors. Przegląd Kardiodiabetologiczny 5: 171-181, 2010 (In Polish).

50. Labuzek K, Kozłowski M, Szkudłapski D, Sikorska P, Kozłowska $M$ and Okopień B: Incretin-based therapies in the treatment of type 2 diabetes - more than meets the eye? Eur J Intern Med 24: 207-212, 2013. 